\title{
Stopping anticoagulant therapy after an unprovoked venous thromboembolism
}

\author{
Clive Kearon MB PhD
}

$\infty \quad$ See related research paper by Rodger and colleagues, page 417

$\mathrm{T}$ hree or 6 months of anticoagulant therapy used to be the standard duration of treatment for almost all patients after a first episode of deep vein thrombosis or pulmonary embolism (collectively referred to as venous thromboembolism). Recently, it has been recognized that the clinical circumstances at the time of the first event strongly influence the risk of a second event after stopping anticoagulant therapy. Therefore, a general recommendation of 3-6 months of anticoagulant therapy is no longer appropriate. ${ }^{1,2}$

If venous thromboembolism is associated with a major nonreversible risk factor such as cancer, patients have a $15 \%$ or greater risk of recurrence in the first year after anticoagulant therapy is stopped. Consequently, patients with active cancer and a first episode of venous thromboembolism usually receive treatment indefinitely. ${ }^{1}$ Conversely, if venous thromboembolism is provoked by a major reversible risk factor, such as recent surgery, the risk of recurrence is about $3 \%$ or lower in the first year if anticoagulants are stopped after 3 months.

Between these 2 extremes lie patients who have had a venous thromboembolism associated with a minor reversible risk factor (e.g., estrogen therapy or soft-tissue leg injury) and those who have had an "unprovoked" venous thromboembolism (also referred to as "idiopathic" or "spontaneous"). ${ }^{3}$ For patients with a minor reversible risk factor, the risk of recurrence is about $5 \%$ in the first year after stopping anticoagulant therapy. ${ }^{3}$ This is considered low enough to justify stopping anticoagulant therapy at the end of 3 months. ${ }^{1}$ However, an unprovoked proximal deep venous thrombosis or pulmonary embolism has a higher risk of recurrence (about 10\% in the first year after stopping therapy). Continuing anticoagulant therapy beyond 3 months confers a greater than $90 \%$ risk reduction for preventing recurrence among these patients; however, if anticoagulants are subsequently stopped after 6 or 12 months of treatment, the risk of recurrence appears to be the same as if anticoagulants had been stopped after 3 months. ${ }^{1}$

This high risk of recurrence is indirect evidence that patients with a first unprovoked proximal deep venous thrombosis or pulmonary embolism should receive anticoagulant therapy indefinitely. Consistent with this, 2 placebo-controlled randomized trials involving patients with unprovoked venous thromboembolism have shown clear benefit from extendedduration anticoagulant therapy with a target international normalized ratio of $2.5^{4}$ or $1.75,{ }^{5}$ compared with stopping therapy at 3-6 months. Both studies were stopped early by their data safety and monitoring committees. ${ }^{4,5}$ Despite the findings of

\section{Key points}

- Patients with a first episode of unprovoked venous thromboembolism have a high risk of recurrence if they stop anticoagulant therapy (about $10 \%$ in the first year).

- Randomized trials support indefinite anticoagulant therapy for these patients if there are no risk factors for bleeding and anticoagulation is well managed.

- The study by Rodger and colleagues in this issue of CMAJ suggests that younger, nonobese women with a first proximal deep venous thrombosis or pulmonary embolism, no signs of post-thrombotic syndrome and a low D-dimer level have a low risk of recurrence and do not require indefinite anticoagulant therapy.

- Patient preference should also be considered in the decision to stop or remain on anticoagulant therapy after 3 months of treatment.

these studies, many physicians are reluctant to extend therapy indefinitely. Reasons for this include concerns that anticoagulant therapy causes more bleeding in the "real world" than in clinical trials and that follow-up in the 2 randomized trials may not have been long enough to accurately reflect the true risk-benefit ratio of indefinite anticoagulant therapy. Also, only half of patients are expected to have a second episode of venous thromboembolism within 10 years of stopping treatment. It is against this background that the results of the study by Rodger and colleagues in this issue of CMAJ should be interpreted. ${ }^{6}$

In this study, patients with a first episode of proximal deep venous thrombosis or pulmonary embolism stopped anticoagulant therapy after 6 months. Rodger and colleagues followed these patients to identify predictors of recurrence and to develop a prediction rule that would identify patients with a low enough risk of recurrence that a long duration of treatment would not be justified. ${ }^{6}$ They found that men had more than twice the risk of recurrence compared with women and that no subgroup of men with a low risk of recurrence could be identified. Younger women who were not obese, had a low D-dimer level at the end of 6 months of therapy and who had no signs of post-thrombotic syndrome had a very low frequency of recurrence. If this combination of factors truly identifies women who can safely stop anticoagulant therapy

Clive Kearon is with Hamilton Health Sciences, Henderson Division, and the Michael G. DeGroote School of Medicine, McMaster University, Hamilton, Ont. 
after 3 or 6 months of treatment for a first unprovoked venous thromboembolism, this is a very important finding.

The study was large and rigorously performed, and it evaluated many potential risk factors for recurrence. However, the factors found to identify women with a very low risk of recurrent venous thromboembolism may represent chance associations rather than true predictors. This is because of the large number of variables evaluated and because continuous variables such as age, body mass index and D-dimer levels were dichotomized using cutoff points selected with knowledge of which patients did or did not have recurrent venous thromboembolism. ${ }^{7}$ On the other hand, because the authors selected variables for the prediction rule using findings from previous studies as well as the associations observed in their own study, the potential for overemphasizing chance associations was reduced.

Consistent with the study by Rodger and colleagues, a lower risk of recurrent venous thromboembolism among women than among men has previously been observed ${ }^{8}$ and has been associated with low D-dimer levels (usually measured after stopping anticoagulation). ${ }^{9}$ While the signs of postthrombotic syndrome are not well-recognized risk factors for recurrent venous thromboembolism, consistency of this finding in men and women in the present study, and previous reports of this association, suggest that the signs of postthrombotic syndrome are valid predictors of the risk of recurrent venous thromboembolism. However, confidence that younger age is a true predictor of reduced risk of recurrence in women is undermined by the simultaneous finding that younger age increased risk in men, coupled with lack of a clear association between age and risk of recurrence in previous studies. Similarly, the inconsistent association of body mass index and risk of recurrence in both men and women questions the validity of this association.

Importantly, the study by Rodger and colleagues confirms that patients who had a first unprovoked venous thromboembolism have a high overall risk of recurrence after stopping anticoagulant (about $11 \%$ at 1 year and about $17 \%$ at 2 years). In addition, they found no association between the presence of residual thrombosis of the deep veins on ultrasound and risk of recurrence, which argues against using this finding to determine the duration of treatment.

Risk of recurrence is not the only factor that determines the optimal duration of anticoagulation therapy for patients with venous thromboembolism. For patients at high risk of bleeding, including those with difficulty maintaining long-term anticoagulant therapy within the therapeutic range, there are arguments against indefinite therapy. Some patients dislike being on anticoagulant therapy, whereas others find it reassuring. ${ }^{10}$
Consequently, patient preference, after the risks and benefits of anticoagulant therapy have been explained, should also influence the decision about the duration of therapy.

In the absence of risk factors for bleeding, which include being older than 75 years, recently published American College of Chest Physician guidelines on antithrombotic therapy strongly recommend that patients with a first episode of proximal deep venous thrombosis or pulmonary embolism remain on indefinite anticoagulant therapy, provided that good anticoagulant monitoring is achievable and indefinite treatment is consistent with patient preferences. ${ }^{1}$ Rodger and colleagues have identified a large subgroup of women who appear to have a low enough risk of recurrence that indefinite anticoagulant therapy would not be indicated. However, as the authors emphasize, the findings of their study need further validation before physicians can confidently recommend stopping anticoagulant therapy to these patients.

Competing interests: None declared. Clive Kearon is chair of the current American College of Chest Physicians Evidence-Based Clinical Practice Guidelines (8th edition) on Antithrombotic Therapy for Venous Thromboembolic Disease.

Acknowledgement: Clive Kearon is supported by the Heart and Stroke Foundation of Ontario.

\section{REFERENCES}

1. Kearon C, Kahn SR, Agnelli G, et al. Antithrombotic therapy for venous thromboembolic disease: American College of Chest Physicians Evidence-Based Clinical Practice Guidelines (8th edition). Chest 2008;133(6 Suppl):454S-545S.

2. Kearon C. Natural history of venous thromboembolism. Circulation 2003;107:122-

3. Baglin $\mathrm{T}$, Luddington $\mathrm{R}$, Brown $\mathrm{K}$, et al. Incidence of recurrent venous thromboembolism in relation to clinical and thrombophilic risk factors: prospective cohort study. Lancet 2003;362:523-6.

4. Kearon C, Gent M, Hirsh J, et al. A comparison of three months of anticoagulation with extended anticoagulation for a first episode of idiopathic venous thromboembolism. N Engl J Med 1999;340:901-7.

5. Ridker PM, Goldhaber SZ, Danielson E, et al. Long-term, low-intensity warfarin therapy for prevention of recurrent venous thromboembolism. N Engl J Med 2003; 348:1425-34.

6. Rodger MA, Kahn SR, Wells PS, et al. Identifying unprovoked thromboembolism patients at low risk for recurrence who can discontinue anticoagulant therapy. CMAJ 2008;179:417-26.

7. Altman DG, Lausen B, Sauerbrei W, et al. Dangers of using "optimal" cutpoints in the evaluation of prognostic factors. J Natl Cancer Inst 1994;86:829-35.

8. McRae S, Tran H, Schulman S, et al. Effect of patient's sex on risk of recurrent venous thromboembolism: a meta-analysis. Lancet 2006;368:371-8.

9. Palareti G, Cosmi B, Legnani C, et al. D-Dimer testing to determine the duration of anticoagulation therapy. N Engl J Med 2006;355:1780-9.

10. Locadia M, Bossuyt PM, Stalmeier PF, et al. Treatment of venous thromboembolism with vitamin $\mathrm{K}$ antagonists: patients' health state valuations and treatment preferences. Thromb Haemost 2004;92:1336-41.

Correspondence to: Dr. Clive Kearon, Hamilton Health Sciences, Henderson Division, 711 Concession St., Hamilton ON L8V 1C3; fax 905 574-7625; kearonc@mcmaster.ca 